\section{Rol de tutor par: experiencia de estudiantes de medicina}

\author{
JESSICA GODOY P. ${ }^{1, \mathrm{a}}$, ALEJANDRA VIDAL V. ${ }^{2}$, \\ MÓNICA ILLESCA P. ${ }^{3, b}$, ELENA ESPINOZA A. ., \\ LORETO FLORES E. ${ }^{4, \mathrm{~d}}$
}

\section{The experience of medical students as peer tutors}

Background: Peer tutoring is a process of accompaniment carried out by a student with certain features and skills, whose objective is to support and guide, academically and emotionally, other students who may require it. Aim: To assess the experience of medical students who played the role of peer tutor. Material and Methods: We carried out semi-structured in-depth interviews, with prior informed consent, to a non-probabilistic and intentional sample of six students who performed the role of peer tutor, during 2017 and 2018. The data analysis followed the scheme of constant comparison and progressive reduction in a manual way, according to the comparative method, guaranteeing scientific rigor, maintaining criteria of credibility, dependence, confirmability, and transferability. Results: The first level identified 234 units of meaning that originated in the third level, two qualitative domains, oriented to the contribution of peer tutors derived from their experience to strengthen both the process of peer tutor training and the management of peer tutoring. Conclusions: Peer tutoring as a teaching-learning strategy contributes to the development of generic competences and metacognitive skills, generating high levels of personal satisfaction and identification their teaching role.

(Rev Med Chile 2021; 149: 765-772)

Key word: Education, Medical; Interdisciplinary Placement; Paired-Associate Learning; Students, Medical.
'Oficina de Educación en Ciencias de la Salud (OFECS). Instituto Enfermería. Facultad de Medicina, Universidad Austral de Chile. Valdivia, Chile.

${ }^{2}$ Oficina de Educación en Ciencias de la Salud (OFECS). Instituto Anatomía, Histología y Patología. Facultad de Medicina, Universidad Austral de Chile. Valdivia, Chile. ${ }^{3}$ Oficina de Educación en Ciencias de la Salud (OFECS), Departamento de Medicina Interna, Facultad de Medicina, Universidad de La Frontera. Temuco, Chile.

${ }^{4}$ Unidad de Apoyo al Aprendizaje de Estudiantes de Pregrado. Universidad Austral de Chile. Valdivia, Chile.

aEnfermera, Magíster en Innovación de la Docencia Universitaria en Ciencias de la Salud, Magíster en Desarrollo Humano. ${ }^{b}$ Enfermera, Doctora en Salud, Magíster en Pedagogía y Gestión Universitaria.

'Profesora de Educación Diferencial, Mención Dificultades de Aprendizaje. dPsicóloga.

Trabajo no recibió financiamiento. Los autores declaran no tener conflictos de interés.

Recibido el 8 de octubre de 2020, aceptado el 1 de febrero de 2021.

Correspondencia a:

Alejandra Vidal V.

Instituto Anatomía, Histología y Patología,

Facultad de Medicina,

Edificio Ciencias Biomédicas, Campus Isla

Teja, Universidad Austral de Chile.

Casilla Postal 567, Valdivia. Chile. avidal@uach.cl
L a tutoría universitaria es una de las experiencias de aprendizaje que acompaña, orienta y $\checkmark$ respalda al estudiantado durante su proceso formativo, apunta al desarrollo de competencias profesionales, personales y académicas ${ }^{1,2}$, considera las dimensiones intelectual, afectiva, personal y social, favoreciendo un desarrollo integral ${ }^{3}$. Se puede definir como una intervención intencionada, sistemática, permanente y formativa, cuyo propósito es apoyar al educando en la construcción de aprendizajes cognitivos, afectivos, socioculturales y existenciales ${ }^{4}$. Implica mayor autonomía por parte del tutorado, por ende, mayor responsabi- lidad para administrar su aprendizaje autónomo ${ }^{2}$. Se considera una actividad extracurricular que constituye un recurso valioso para mejorar los resultados académicos de los estudiantes, tendiente a reducir la reprobación, el rezago y la deserción académica ${ }^{5}$.

Desde la perspectiva docente, promueve la comunicación asertiva, identifica factores de riesgo y protección, fortalece la convivencia y la conformación de la identidad de los tutorados ${ }^{4}$, propiciando su autoevaluación, autorregulación y autodeterminación, potenciando el desarrollo de habilidades metacognitivas ${ }^{3,6}$, así, el estudiante 
asume la responsabilidad de desarrollar sus propias habilidades ${ }^{7}$.

En este contexto, una de las estrategias utilizadas por la gran mayoría de las universidades son las tutorías entre pares, destacándose como un poderoso medio para mejorar la calidad, pertinencia y equidad del proceso educativo ${ }^{8}$. Surgen como una forma de dar respuesta a nuevas necesidades de la universidad y estudiantado, a través de una atención más personalizada, capaz de asumir la diversidad y multiculturalidad ${ }^{9}$, permitiendo que los educandos se adapten de mejor forma, aumenten su rendimiento y se desarrollen en el ámbito académico y profesional ${ }^{1}$.

Es una estrategia para el aprendizaje, en la que estudiantes mediante la congruencia cognitiva y social orientan a sus compañeros para que logren condiciones óptimas para aprender, se enfocan hacia asignaturas con mayor índice de reprobación o alumnos con indicadores de riesgos, tanto académicos como socioculturales ${ }^{10}$. Las tutorías pueden agruparse en tres: académica (apoyo a los procesos de enseñanza-aprendizaje); curricular (orientación para elegir itinerario académico y profesional) y personal (asesoría respecto al desarrollo integral $)^{11}$.

El tutor par tiene la responsabilidad de lograr aprendizajes en sus tutorados, sin implicancias curriculares o evaluativas, su experiencia como estudiante la utiliza para orientar a sus compañeros sobre cómo abordar situaciones problemáticas. La dinámica grupal es asimétrica y está determinada por la distribución de responsabilidades ${ }^{12}$. Además, deben apoyar los procesos madurativos, favorecer la actitud crítica, autonomía, toma de decisiones, propiciar autoconocimiento, autorregulación y fortalecer la autoestima de sus tutorados ${ }^{4,13}$.

Esta estrategia tiene beneficios tanto para el tutorado como para el tutor par. En relación a este último, es posible identificar: adquisición de competencias propias de la disciplina, mayor seguridad y habilidades comunicativas ${ }^{14}$, desarrollo y consolidación de su base de conocimientos, ganancia de experiencia docente, satisfacción asociada a enseñar, alcanzando aprendizajes sobre distintos ámbitos ${ }^{15}$; logran confianza académica y personal, compensación a través de un aporte económico o créditos para los títulos, mejoran su gestión del tiempo y habilidades de comunicación, a través, de la relación con sus tutorados, comprenden los procesos pedagógicos y empatizan con el rol docente, evalúan sus propias limitaciones ${ }^{16}$. En tanto, los tutorados refuerzan hábitos de estudio, mejoran el rendimiento académico, adquieren motivación y potencian las relaciones sociales ${ }^{15}$. Sumado a ello, está demostrado que los ambientes favorables de trabajo fortalecen el desarrollo de competencias genéricas ${ }^{17}$.

Para los tutores de medicina, la tutoría entre pares permite el desarrollo de competencias comunicativas fomentando la capacidad de escucha y argumentación, refuerza el conocimiento previo, adquisición de experiencia en docencia y compromiso, lo que permite fortalecer valores como la solidaridad ${ }^{18}$.

El estudiante tutor necesita compañía y guía de un profesor para adquirir bases y herramientas tutoriales ${ }^{19}$ y pedagógicas que le permitan motivar la participación de los tutorados y facilitar la dinámica socio-cognitiva que se genera en el grupo $^{20}$. Requiere competencias de comunicación efectiva para apoyar a sus tutorados en el desarrollo de estrategias para la resolución de conflictos $^{21}$. Debe ser reflexivo para identificar, conocer y comprender las prácticas de aprendizaje de su grupo, analizar las propias con un espíritu de mejora continua ${ }^{19}$, cultivar interés por sus tutorados, tener capacidad de observación, respeto, iniciativa, compromiso, objetividad, flexibilidad, confianza, empatía, mediación y escucha activa libre de prejuicios ${ }^{3}$.

Lo anterior refleja la importancia de un programa formal de capacitación dirigido a los estudiantes para desempeñar el rol de tutor par. Existe evidencia que mejora significativamente el aprendizaje cooperativo y la organización del aprendizaje $\mathrm{e}^{22}$.

Al respecto, la Escuela de Medicina de la Universidad Austral de Chile (UACh), no ajena a estas directrices, se ha sumado a la experiencia de tutor par, donde estudiantes de cursos superiores orientan y asesoran a compañeros como acción complementaria a las actividades curriculares. Son de carácter grupal, con un máximo de 8 estudiantes por grupo, incluyen tres aspectos: materia, práctica y asesoramiento personal, donde se abordan temas vistos en clase, trabajando habilidades y destrezas vinculadas a competencias profesionales y derivación para atención psicológica o psicopedagógica si detecta que su par necesita apoyo. Este Programa de Tutores Pares y acompañamiento estudiantil se 
inició en el año 2014, y desde el 2017 se encuentra a cargo de la Oficina de Educación en Ciencias de la Salud (OFECS), respondiendo a uno de sus ejes estratégicos vinculados a apoyo estudiantil.

En el contexto de la investigación, la selección de tutores pares se realiza según antecedentes académicos, habilidades sociales y disposición para enseñar, y corresponde a estudiantes que cursan $4^{\circ}$ o $5^{\circ}$ año de medicina. La preparación para desarrollar este rol se genera a través de talleres organizados por la OFECS, considerando el área cognitiva, procedimental y actitudinal, abordando temas como: rol del tutor, estilos de aprendizaje, estrategias de enseñanza, manejo del tiempo, planificación tutorial y habilidades interpersonales como comunicación efectiva y empatía. El equipo encargado está conformado por dos académicas, una psicóloga y una profesora de educación diferencial, sistemáticamente realizan reuniones con el fin de acompañar, evaluar el desarrollo del proceso tutorial y responder inquietudes para tomar acciones oportunamente.

La evaluación de los tutores pares con respecto a su participación en este programa ha sido muy bien valorada como una instancia de aprendizaje integral, sin embargo, no existe evidencia cualitativa por parte del propio sujeto en el contexto en el que se desenvuelve, donde la postura que asume frente a la realidad, le devuelve la responsabilidad sobre sus acciones y decisiones. Surge así la interrogante ¿cuál es el significado que le otorgan los tutores pares a la experiencia vivida? En este escenario, se planteó como objetivo general: conocer la experiencia de los estudiantes de la carrera de medicina que desempeñaron el rol de tutor par. Los objetivos específicos se orientaron a indagar en relación con las acciones utilizadas para desempeñar su rol, descubrir los factores que inciden en la experiencia, develar el desarrollo de competencias adquiridas y explorar sugerencias para potenciar este tipo de práctica educativa.

\section{Material y Método}

Investigación educativa, exploratoria, descriptiva, bajo el paradigma cualitativo mediante un estudio intrínseco de $\operatorname{casos}^{23}$.

La muestra fue no probabilística e intencionada de casos por criterios $^{24}$, constituida por 6 informantes claves voluntarios de sexo masculino y 2 de sexo femenino, quienes cumplieron los siguientes criterios de inclusión: haber recibido la capacitación de tutor par de la OFECS, desempeñar el rol durante los años 2017 y 2018, al menos un semestre de forma ininterrumpida, y aceptar participar voluntariamente en la investigación mediante firma del consentimiento informado.

La recolección de los datos se realizó en el segundo semestre del año 2017 y primer semestre del 2018, a través, de la técnica de entrevista en profundidad semiestructurada ${ }^{25}$, utilizando una pauta con preguntas orientadoras, enmarcadas en el contexto de los objetivos de la investigación.

La información fue recogida a través de una grabadora de sonido y notas de campo, previa autorización y firma del consentimiento informado. Se realizó la transcripción literal de la entrevista con el fin de cautelar la rigurosidad metodológica. Los datos se recopilaron hasta llegar al punto de saturación, cabe señalar que el proceso de recogida y análisis de datos se hizo de forma simultánea ${ }^{26}$.

El análisis de los datos se realizó siguiendo el esquema de comparación constante y reducción progresiva de forma manual de acuerdo con el método comparativo de constante ${ }^{27}$, estableciendo categorías de datos, siendo comparadas durante todo el proceso de análisis. Se determinaron 3 niveles, reducción de datos, presentación de estos, diseño y verificación de conclusiones ${ }^{28}$.

- Nivel 1: identificación y segmentación de narraciones textuales en relación a un tema, para agrupar en categorías descriptivas, estableciendo unidades de significado.

- Nivel 2: construcción de metacategorías, a través de la identificación de similitudes estructurales y elementos comunes de las categorías descriptivas.

- Nivel 3: identificación de dominios cualitativos, este proceso se realizó de forma simultánea al categorizar, ya que al agrupar las unidades paralelamente se fueron reduciendo a un solo significado.

La confiabilidad se garantizó utilizando los criterios de rigor determinados por las estrategias de veracidad: valor de verdad o credibilidad; dependencia o consistencia, aplicabilidad o transferibilidad; y neutralidad o confirmabilidad ${ }^{29}$.

El estudio consideró los 7 requisitos éticos de investigación ${ }^{30}$; valor social: contribuye a generar 
conocimientos para optimizar el Programa Escuela de Tutores; validez científica: mediante un diseño riguroso, cuyos productos, además, fueron triangulados por investigadores; selección equitativa del sujeto: se seleccionaron los informantes claves según las interrogantes del estudio, sin ocasionarles daños directos; proporción favorable de riesgo-beneficio: se cumplió respetando los principios de beneficencia y no maleficencia, sin conflicto alguno para los participantes; evaluación independiente: aprobación del Comité de Ética de la Facultad de Medicina de la UACh (13 de julio de 2017); incluyó una participación voluntaria, firma del consentimiento informado, se cauteló el respeto a los sujetos mediante la posibilidad de retirarse, garantizando el anonimato y la confidencialidad de la información.
Resultados

\section{Nivel 1}

Se identificaron 234 unidades de significado relevantes para el estudio, las que fueron agrupadas en 6 categorías descriptivas codificadas en función de conocer la opinión de los estudiantes tutores con respecto a la experiencia tutorial (Tabla 1).

\section{Nivel 2}

Representa los núcleos temáticos emergentes o metacategorías que surgieron del nivel 1. Posterior a un proceso de comparación intercategorías en el que se buscaron similitudes estructurales y elementos comunes, se identificaron 3 metacategorías, en las cuales se reagruparon todas las categorías (Tabla 2).

Tabla 1. Distribución de frecuencias de las unidades de significado de las categorías descriptivas codificadas con respecto al objeto de estudio

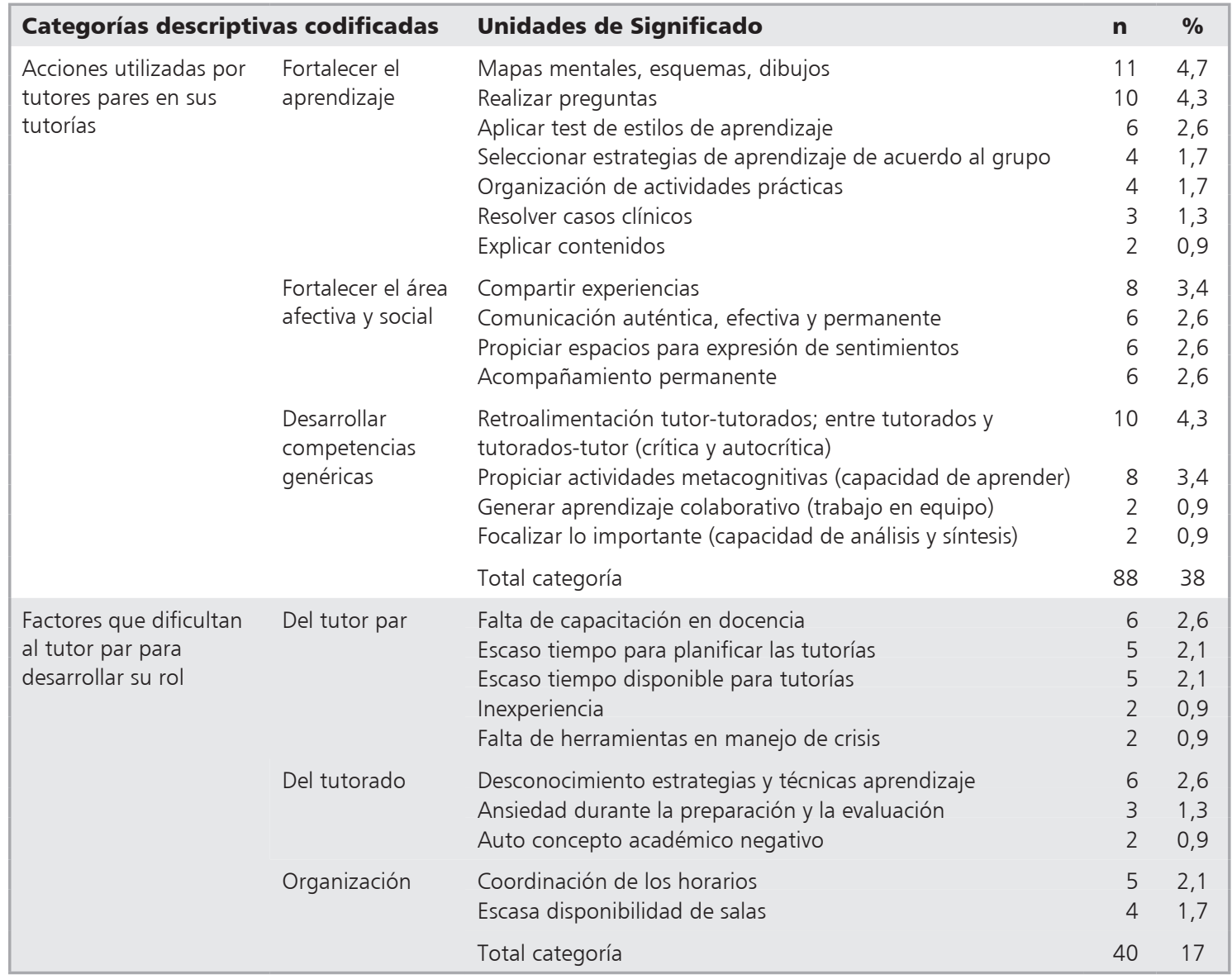


Tutor par: experiencia de estudiantes - J. Godoy et al

\begin{tabular}{|c|c|c|c|c|}
\hline \multirow{8}{*}{$\begin{array}{l}\text { Competencias genéricas } \\
\text { desarrolladas a través } \\
\text { del rol tutor par }\end{array}$} & \multirow[t]{4}{*}{ Interpersonal } & Habilidades interpersonales & 4 & 1,7 \\
\hline & & $\begin{array}{l}\text { Capacidad de crítica y autocritica (Desarrollo procesos } \\
\text { metacognitivos) }\end{array}$ & 5 & 2,1 \\
\hline & & Responsabilidad social & 3 & 1,3 \\
\hline & & Capacidad de trabajar en equipo interdisciplinar & 2 & 0,9 \\
\hline & \multirow[t]{2}{*}{ Sistémica } & Capacidad de aprender & 9 & 3,8 \\
\hline & & Capacidad de aplicar conocimientos a la práctica & 4 & 1,7 \\
\hline & \multirow[t]{2}{*}{ Instrumental } & Comunicación oral y escrita & 8 & 3,4 \\
\hline & & Total categoría & 35 & 15 \\
\hline \multirow{9}{*}{$\begin{array}{l}\text { Factores que facilitan } \\
\text { al tutor par para } \\
\text { desarrollar su rol }\end{array}$} & \multirow[t]{4}{*}{ Del tutorado } & Motivación para aprender & 6 & 2,6 \\
\hline & & Asistencia a tutorías & 3 & 1,3 \\
\hline & & Estudio previo de los temas & 3 & 1,3 \\
\hline & & Confianza con el tutor par & 2 & 0,8 \\
\hline & \multirow[t]{3}{*}{ Del tutor par } & Cercanía con los tutorados & 6 & 2,6 \\
\hline & & Preparación de material para las tutorías & 3 & 1,3 \\
\hline & & Reprobación de asignatura & 2 & 0,8 \\
\hline & \multirow[t]{2}{*}{ Organización } & Recursos materiales & 2 & 0,8 \\
\hline & & Total categoría & 27 & 11 \\
\hline \multirow{8}{*}{$\begin{array}{l}\text { Sugerencias del tutor } \\
\text { par para optimizar el } \\
\text { proceso tutorial }\end{array}$} & \multirow{4}{*}{$\begin{array}{l}\text { Capacitación } \\
\text { tutor par }\end{array}$} & Intervención en crisis & 6 & 2,6 \\
\hline & & Habilidades sociales & 4 & 1,7 \\
\hline & & Comunicación & 3 & 1,3 \\
\hline & & Planificación de tutorías & 2 & 0,8 \\
\hline & $\begin{array}{l}\text { Capacitación del } \\
\text { tutorado }\end{array}$ & Técnicas de estudio & 2 & 0,8 \\
\hline & Organización & Disponibilidad de salas & 3 & 1,3 \\
\hline & \multirow[t]{2}{*}{ Gestión } & Motivación a los tutorados a participar & 3 & 1,3 \\
\hline & & Total categoría & 23 & 10 \\
\hline \multirow{4}{*}{$\begin{array}{l}\text { Aportes de la } \\
\text { experiencia al proceso } \\
\text { formativo del tutor par }\end{array}$} & $\begin{array}{l}\text { Funciones de la } \\
\text { medicina }\end{array}$ & $\begin{array}{l}\text { Motivación para enseñar } \\
\text { Identificación con el ejercicio del rol docente }\end{array}$ & $\begin{array}{l}8 \\
6\end{array}$ & $\begin{array}{l}3,4 \\
2,6\end{array}$ \\
\hline & \multirow[t]{3}{*}{ Ámbito personal } & Satisfacción personal & 7 & 3,0 \\
\hline & & Total categoría & 21 & 9 \\
\hline & & Total & 234 & 100 \\
\hline
\end{tabular}

Tabla 2. Distribución porcentual entre metacategorías, categorías descriptivas codificadas y unidades de significado con respecto al objeto de estudio

\begin{tabular}{|c|c|c|c|}
\hline \multirow[t]{2}{*}{ Metacategoría } & \multirow[t]{2}{*}{ Categorías descriptivas codificadas } & \multicolumn{2}{|c|}{$\begin{array}{l}\text { Unidades de } \\
\text { significado }\end{array}$} \\
\hline & & $\mathbf{n}$ & $\%$ \\
\hline $\begin{array}{l}\text { Estrategias utilizadas por el } \\
\text { tutor par }\end{array}$ & Acciones utilizadas por tutores pares en sus tutorías & 88 & 38 \\
\hline $\begin{array}{l}\text { Beneficios del ejercicio de } \\
\text { tutor par }\end{array}$ & $\begin{array}{l}\text { Competencias genéricas desarrolladas a través de la práctica del rol tutor par } \\
\text { Sugerencias del tutor par para optimizar el proceso tutorial } \\
\text { Aportes de la experiencia al proceso formativo del tutor par }\end{array}$ & $\begin{array}{l}35 \\
23 \\
21\end{array}$ & $\begin{array}{r}15 \\
10 \\
9\end{array}$ \\
\hline \multirow[t]{2}{*}{$\begin{array}{l}\text { Factores que inciden en el } \\
\text { ejercicio del rol de tutor par }\end{array}$} & $\begin{array}{l}\text { Factores que obstaculizan la labor del tutor par } \\
\text { Factores que facilitan la labor del tutor par }\end{array}$ & $\begin{array}{l}40 \\
27\end{array}$ & $\begin{array}{l}17 \\
11\end{array}$ \\
\hline & Total & 234 & 100 \\
\hline
\end{tabular}




\section{Nivel 3}

Después del análisis secuencial y transversal de las metacategorías, surgen dos dominios cualitativos emergentes que reflejan lo expresado por las(os) participantes del estudio.

1. Aportes de tutores pares derivados de su experiencia para fortalecer el proceso de formación de futuros tutores pares. Representa la opinión de los estudiantes en relación con los beneficios del ejercicio del rol de tutor par y a las estrategias utilizadas por este.

2. Contribución de tutores pares derivados de su experiencia para fortalecer la gestión de las tutorías de pares. Evidencia la valoración del estudiantado con respecto a los factores que inciden en el ejercicio del rol de tutor par.

\section{Discusión}

Con respecto a las acciones utilizadas por tutores pares en sus tutorías se perfilan en tres ámbitos de acción, orientadas a fortalecer aprendizajes (mapas mentales, preguntas, casos clínicos, entre otros), desarrollar competencias genéricas y fortalecer el área afectiva y social, lo que coincide con lo descrito en la literatura ${ }^{2,4}$. Consideran significativo mantener una retroalimentación bidireccional constante para el desarrollo de competencias genéricas, propiciando la reflexión con respecto a sus propias prácticas ${ }^{19}$, fomentando el trabajo en equipo ${ }^{6}$. Especial importancia otorgan a la comunicación efectiva y permanente para fortalecer el área afectiva y social, a través de compartir experiencias, propiciar la expresión de sentimientos y acompañamiento, lo que concuerda con la evidencia encontrada ${ }^{3,4,18,19}$.

Los resultados de este estudio evidencian que el ejercicio del rol como tutor par propicia el desarrollo de competencias genéricas: sistémicas (capacidad de aprender y aplicar conocimientos a la práctica), interpersonales (capacidad de crítica y autocrítica, habilidades interpersonales, responsabilidad social y capacidad de trabajar en equipo interdisciplinar) e instrumentales (comunicación oral y escrita), lo que coincide con diversos autores $^{3,14,16,18}$. La valoración que otorgan a esta experiencia trasciende como un aporte real a su proceso formativo, en un escenario extracurricular donde asumen responsabilidades del propio aprendizaje y de otros. Asimismo, destacan la satisfacción personal, resultado del vínculo con sus tutorados, y que actúa como fuente de motivación para enseñar y entregar su mejor esfuerzo, identificándose con el rol docente y vislumbrando el ejercicio de la docencia una vez que concluyan su formación de pregrado $^{14,16}$.

Se develan factores facilitadores y obstaculizadores que inciden en el rol como tutor par, asociados al "tutor par", "tutorados" y "organización", siendo los obstaculizadores los más identificados. La falta de capacitación en docencia, escaso tiempo para planificar las tutorías y disponibilidad para realizarlas, inexperiencia y falta de herramientas para el manejo de crisis, es coincidente con otros estudios $^{12,20-21}$. En tanto, les facilita su rol la cercanía con los tutorados, la preparación de material y experiencia de haber reprobado ${ }^{15,21}$.

Con respecto al tutorado, los factores que les dificultan se relacionan con el desconocimiento de estrategias y técnicas de aprendizaje, ansiedad previa y durante las evaluaciones y autoconcepto académico negativo, lo que refuerza la necesidad de que el tutor par cuente con capacitación para apoyar los procesos madurativos, favorecer la actitud crítica, autonomía, toma de decisiones, propiciar el autoconocimiento, autorregulación y fortalecer la autoestima de sus tutorados ${ }^{13}$. En relación con los factores del tutorado que facilitan su rol, identificaron la motivación para aprender, la asistencia a las tutorías, estudio previo de los temas y confianza con el tutor, lo que es coincidente con otros estudios ${ }^{15,16}$.

Los factores que dificultan (coordinación de los horarios, disponibilidad de salas) y favorecen (recursos materiales) su rol como tutor par con respecto a la organización, evidencia la importancia de la gestión que pueda realizar el equipo encargado, con el fin de optimizar la experiencia.

En cuanto a las sugerencias para optimizar el proceso tutorial, se identificaron: fortalecer la capacitación de tutores para el manejo de situaciones de crisis, puntualmente contención, comunicación efectiva y planificación de las tutorías, si bien, el programa de tutores pares incluye capacitaciones en las temáticas señaladas, queda de manifiesto la necesidad de fortalecerlas, desarrollándolas de una manera vivencial y práctica, evidenciando la importancia de un programa formal de capacitación dirigido a los tutores, para el buen ejercicio de su $\mathrm{rol}^{22}$.

El valor del proceso tutorial radica en que brin- 
da a los tutores/as la posibilidad de aprender desde el ejercicio del rol, de manera vivencial, les permite potenciar competencias genéricas interpersonales y sistémicas e instrumentales, propicia habilidades metacognitivas, fortaleciendo sus conocimientos y desarrollando herramientas para apoyar de manera efectiva a sus tutorados/as, ya que el proceso es tremendamente satisfactorio desde el punto de vista personal, transformándose en una experiencia relevante en su proceso formativo.

Agradecimientos: A los tutores y tutoras pares por su participación en las entrevistas.

\section{Referencias}

1. Álvarez-Pérez P. La función tutorial del profesorado universitario: una nueva competencia de la labor docente en el contexto del Espacio Europeo de Educación Superior. Rev Port Pedagog 2014; 47(2): 85-106. Disponible en: https://www.researchgate.net/publication/308330960 [consultado el 5 de enero de 2020].

2. Pérez Cusó F J, González Lorente C, González Morga N , Martínez Juárez M. Tutoría en la Universidad: un estudio de caso en la Facultad de Educación de la Universidad de Murcia. Educ XX1 2017; 35: 91-110. Disponible en: https://revistas.um.es/educatio/article/ view/298531 [consultado el 5 de enero de 2020].

3. Álvarez M, Dorio I, Figuera P, Fita E, Forner A, Martínez A, Torrado M. La acción tutorial en la universidadEl perfil de los estudiantes universitarios. Implicaciones para la acción tutorial. En Rodríguez Espinar R, coordinador. Manual de tutoría universitaria. Recursos para la acción. Ediciones Octaedro. Barcelona 2012. p. 20-36.

4. Obaya A, Vargas Y. La tutoría en la educación superior. Investigación Educativa. Educ Química 2014; 25 (4): 478-87. Disponible en: https://www.sciencedirect.com/ science/article/pii/S0187893X14700709, [consultado el 8 de enero de 2020].

5. Hervás M, Fernández F. D, Arco J L y Miñaca M I. Efectos de un programa de Aprendizaje-Servicio en el alumnado universitario. Rev Electron Investig Psicoeduc Psigopedag 2017; 15(41): 126-46. Disponible en: https:// www.redalyc.org/pdf/2931/293150349005.pdf [consultado el 20 de junio de 2020].

6. Lobato C, Celia M. La Orientación y Tutoría universitaria: una aproximación actual. Red U 2013; 11 (2): 17-25. Disponible en: https://polipapers.upv.es/index. php/REDU/article/view/5564 [consultado el 8 de enero de 2020].
7. Murdoch-Eaton D, Whittle S. Generic skills in medical education: developing the tools for successful lifelong learning. Med Educ 2012; 46: 120-8. Disponible en: https://pubmed.ncbi.nlm.nih.gov/22150203/ [consultado el 15 de enero de 2020].

8. Narro Robles J, Arredondo Galvan M. La tutoría: Un proceso fundamental en la formación de los estudiantes universitarios. Perfiles Educ 2013; 35 (141): 132-51. Disponible en: http://www.scielo.org.mx/scielo.php?pi$\mathrm{d}=$ S0185-26982013000300009\&script $=$ sci_abstract [consultado el 21 de junio de 2020].

9. Álvarez M, Álvarez J. La tutoría universitaria: del modelo actual a un modelo integral. Rev Electron Interuniv Form Profr 2015; 18 (2): 125-42. Disponible en: https:// revistas.um.es/reifop/article/view/219671 [consultado el 8 de enero de 2020].

10. Fior C. Contribuições da monitoria e da tutoria entre pares para a permanência do estudante no ensino superior: Análise de publicações do CLABES de 2011 a 2014, CCLA 15 nov 2017. Disponible en: https://revistas.utp. ac.pa/index.php/clabes/article/view/1584 [consultado el 15 de enero de 2020].

11. Solaguren-Beascoa M, Moreno L. Escala de actitudes de los estudiantes universitarios hacia las tutorías académicas. Educ XX1 2016; 19(1): 247-66. Disponible en: https://www.redalyc.org/pdf/706/70643085011.pdf [consultado el 15 de enero de 2020].

12. Menéndez JL. El problema terminológico de la tutoría entre iguales y la afirmación de su especificidad didáctica. Observar 2010; 4: 66-94. Disponible en: https://web. ua.es/es/ice/documentos/tutorial/material/ivjornada/ la-tutoria-entre-iguales.pdf. [consultado el 15 de enero de 2020].

13. Sobrado L. Plan de acción tutorial en los centros docentes universitarios: el rol del profesor tutor. Rev Interuniv Form Profr 2008; 22 (1): 89-108. Disponible en: https:// dialnet.unirioja.es/servlet/articulo? codigo $=2541041$ [consultado el 8 de enero de 2020].

14. Bravo A, Faúndez C, Moraga F, Borzone M. Formación de Estudiantes Tutores: Un Apoyo Fundamental para Potenciar el Desarrollo de Prácticas de Física. Form Univ 2019; 12 (2): 63-72. Disponible en: https:// scielo.conicyt.cl/scielo.php?script=sci_arttext\&pi$\mathrm{d}=$ S0718-50062019000200063\&lng=es\&nrm=iso [consultado el 8 de enero de 2020].

15. Vaughan B, Macfarlane C. Perceived teaching quality between near-peer and academic tutors in an osteopathic practical skills class. Int J Osteopath Med 2015; 18 (3): 219-29. Disponible en: https://www.journalofosteopathicmedicine.com/article/S1746-0689(15)00046-2/ fulltext [consultado el 20 de mayo de 2020]. 
16. Nisbet J, Haw M, Fletcher A. The role of tutors in peer led teaching. Educ Chem Eng 2014; 9(1): 15-9. Disponible en: https://www.sciencedirect.com/science/article/ pii/S1749772813000171?via\%3Dihub\#! [consultado el 8 de enero de 2020].

17. Natoli R, Jackling B, Seelanatha L. The impact of instructor's group management strategies on students' attitudes to group work and generic skill development. Pedagogies 2014; 9 (2): 116-32. Disponible en: https://www.tandfonline.com/doi/abs/10.1080/155448 0X.2014.912519 [consultado el 20 de enero de 2020].

18. Torrado-Arenas DM, Manrique-Hernández E, Ayala-Pimentel J. La tutoría entre pares: una estrategia de enseñanza y aprendizaje de histología en la Universidad Industrial de Santander. MÉD UIS 2016; 29 (1): 71-5. Disponible en: https://revistas.uis.edu.co/index.php/ revistamedicasuis/article/view/5490/5707 [consultado el 20 de enero de 2020].

19. Cardozo-Ortíz CE. Tutoría de pares como una estrategia pedagógica universitaria. Educ Educ 2011; 14 (2): 309-25. Disponible en: http://www.scielo.org.co/pdf/ eded/v14n2/v14n2a05.pdf [consultado el 15 de enero de 2020].

20. Slavin RE. Research on Cooperative Learning and Achievement: What We Know, What We Need to Know. Contemp Educ Psychol 1996; 21 (1): 43-69. Disponible en: https://www.sciencedirect.com/science/article/abs/ pii/S0361476X96900041?via\%3Dihub [consultado el 15 de enero de 2020].

21. Savickas ML, Nota L, Rossier J, Dauwalder JP, Duarte M, Guichard J, et al. Life designing: A paradigm for career construction in the 21st century. J Vocat Behav 2009; 75(3): 239-50. Disponible en: https://www.researchgate.net/publication/222013431_Life_designing_A_ paradigm_for_career_construction_in_the_21st_century [consultado el 10 de marzo de 2020].

22. Shiozawaa T, Hirt B , Lammerding-Koeppel M. The influence of tutor training for peer tutors in the dissection course on the learning behavior of students. Ann Anat 2016; 208: 212-6. Disponible en: https://www.science-
direct.com/science/article/pii/S0940960216301273?casa_token=ALsaC4ssPvkAAAAA:Z--U5tMsKUI4bPsF4dS1yFNw_SnTNJA314DlwaGkVmc1tbXGLkGYO57VtOU0KIlO4fo_A8LieUKN [consultado el 9 de abril de 2020].

23. Stake R. Investigación con estudio de casos. $2^{\mathrm{a}}$ ed. Madrid: Ediciones Morata, S.L.; 1999. p. 15-23.

24. Polit D., Hungler B. Investigación Científica en Ciencias de la Salud. $6^{\circ}$ ed. México: Mc Graw- Hill Interamericana; 2000. p. 525-45.

25. Fernández R, Fernández C, Baptista M. Metodología de la Investigación. 5a ed. México: Mc Graw-Hill Interamericana; 2010. p. 418. Disponible en: https://www.esup. edu.pe/descargas/dep_investigacion/Metodologia $\% 20$ de\%20la\%20investigaci\%C3\%B3n\%205ta\%20Edici\%C3\%B3n.pdf. [consultado el 10 de enero de 2020].

26. Martínez-Salgado C. El muestreo en investigación cualitativa. Principios básicos y algunas controversias. Cien Saude Colet 2012; 17 (3): 613-9. Disponible en: https://www.scielo.br/scielo.php?script=sci_arttext\&pid=S1413-81232012000300006\&lng=es\&tlng=es. [consultado el 10 de enero de 2020].

27. Glaser BG, Strauss AL. El método de comparación constante de análisis cualitativo. In: The discovery of Grounded Theory: strategies for qualitative research. New York: Aldine; 1967. p. 101-15.

28. Miles M, Huberman M. Qualitative Data Analysis: An Expanded Sourcebook. 2a ed. California: SAGE Publications, Inc; 1994. p. 1-15. Disponible en: https:// vivauniversity.files.wordpress.com/2013/11/milesandhuberman1994.pdf [consultado el 10 de enero de 2020].

29. Guba E, Lincoln Y. Effective evaluation: Improving the usefulness of evaluation results through responsive and naturalistic approaches. San Francisco: Jossey-Bass; 1981.

30. Lolas-Steke F, Quezada-Sepúlveda A. Pautas éticas de investigación en sujetos humanos: nuevas perspectivas. Santiago de Chile: Programa Regional de Bioética OPS/ OMS; 2003. p. 83-93. 